\title{
Collaboration is key to strengthening surgical research capacity in sub-Saharan Africa
}

\section{A call for more research in areas with the highest burden of disease}

Global health research efforts are not being instigated in countries that have the highest burden of disease or the greatest clinical need. ${ }^{[1,2]}$ The so-called ' $10 / 90$ gap' is well known, describing an estimated $10 \%$ of global health research devoted to conditions that account for $90 \%$ of the global disease burden. While much effort has addressed this disparity in the past 25 years, recent research from the Lancet Commission on Global Surgery indicates that high-income countries still account for $85 \%$ of published articles from the leading 35 countries undertaking surgical research. ${ }^{[3]}$ This situation needs to change. Disease characteristics and research findings from developed countries are potentially impractical and misleading for clinicians practising in low- and middle-income countries (LMICs) that are less well resourced.

Sub-Saharan Africa (SSA) is facing a quadruple burden of disease. Diseases of lifestyle, impoverishment, and HIV and trauma epidemics place massive strains on our healthcare systems. Women in Africa are developing breast cancer on average 15 years earlier than women in North America and Europe. This early onset of breast cancer in Africa suggests different risk factors and a different disease profile. ${ }^{[4]}$ However, full understanding of surgical disease and best treatment practices in LMICs are restricted by an absence of local research on local areas of need. ${ }^{[3]}$ Many other critically important local surgical questions have yet to be thoroughly addressed.

Surgical trainees and medical officers are the workforce addressing the surgical needs of this burden. They have to cope with an extremely pressured working environment, time constraints, lack of research training and a surgical culture that leans towards service delivery over training or research. This has meant that there is a paucity of locally relevant research output from our surgical trainees, particularly in comparison with our overseas counterparts.

The Health Professions Council of South Africa recently enforced a recommendation of the College of Surgeons of South Africa to make the MMed degree mandatory prior to acceptance on the specialist registry as a general surgeon. This stimulus, while commendable, will fail to encourage new research capacity if it is not accompanied by good departmental supervision and support in timely pursuit of relevant questions. It is up to the universities and surgical institutions to address the fact that lack of supervision, skills and time to pursue research still persist. Further obstacles to fostering a research-friendly culture include poor patient record keeping with the absence of good-quality clinical databases, a lack of accessible data-capturing systems in most training institutions, and little institutional memory or impetus for clinical research or audit. Lastly, there is a poor track record of collaborative research in southern African surgical departments, most having preferred insular, single-centre research.

\section{Surgical research collaboratives provide a solution}

In the UK over the past 7 years, trainee-led regional networks in general surgery have been developed to adopt a novel collaborative approach to research. Collaboration between trainees in several hospitals allows for a larger number of patients to be included in studies over a shorter time, prevents repetition, and makes the results more applicable than those arising from single-centre studies. Trainees are ideally placed to deliver this model; they follow a rotational pattern through several hospitals, are in regular contact with each other, are motivated, and are expected to produce evidence of research and audit.

The first regionally developed general surgical research collaborative was the West Midlands Research Collaborative. Their published randomised controlled trial, ROSSINI (Reduction Of Surgical Site Infection using a Novel Intervention), ${ }^{[5]}$ recruited 760 patients from 21 centres to use either a wound-edge protection device or standard practice. The rapid recruitment (the trial ran ahead of schedule throughout) and minimal loss to followup demonstrated the ability of trainees to plan and conduct high-quality multicentre research. Other regional and specialty-based collaboratives were subsequently established, allowing for almost complete coverage of the UK. In general surgery, these regional networks recently delivered the Multicentre Appendicectomy Audit that included 3326 consecutive patients undergoing appendicectomy from 95 centres in just 2 months! $!^{[6]}$ Further trainee-led randomised trials and national cohort studies are ongoing, and are recruiting patients from across the UK. ${ }^{[7]}$

The hands-on engagement of trainees in research and audit projects has obvious educational benefits. With this in mind, the collaborative model has now been expanded to include medical students across the UK and Ireland through the Student Audit and Research in Surgery network (STARSurg, www.starsurg.org). This is now in its third year, and the most recent cohort study saw over 1000 collaborators collect data on over 9200 patients across 168 hospitals. Its first cohort study, investigating the impact of postoperative non-steroidal antiinflammatory drugs on adverse events after gastrointestinal surgery, was published in the British Journal of Surgery. ${ }^{[8]}$

Furthermore, these collaborations have started to publish under a group name in order to flatten the traditional hierarchies associated with academic authorship and provide academic recognition as an added incentive to participation. Ultimately, it is hoped that as these undergraduate and postgraduate collaborators progress to become consultants, a culture of research, audit and trials will be embedded in their surgical practice.

\section{Some collaborative success to date in SSA}

The South African Surgical Outcomes Study was a 7-day national, multicentre, prospective, observational cohort study of all patients older than 16 years of age undergoing inpatient non-cardiac surgery between 19 and 26 May 2014 at 50 government-funded hospitals in South Africa (SA). This collaborative effort recruited 3927 patients from 45 hospitals located throughout the country and was recently published in the SAMJ. ${ }^{[9]}$

Hospitals throughout the world providing emergency surgical care were invited to collaborate in GlobalSurg. ${ }^{[10]}$ This was a multicentre, international, prospective cohort study that aimed to determine universal processes related to best outcome in emergency abdominal surgery over a 2-week period during July - November 2014. ${ }^{[10]}$ Over 350 centres, including 15 from SSA, contributed to this truly international evaluation.

\section{A way forward}

The remarkable success in the UK with collaborative networks and these recent local accomplishments may suggest that a simi- 
lar model in SSA could provide the stimulus to increase research capacity, particularly in the surgical outcomes and quality improvement domains. ${ }^{[11]}$ All collaboratives from the UK have found alignment with existing organised structures beneficial in providing advice and support, including professional specialty associations. In SA, for example, the Association of Surgeons of South Africa, the Surgical Research Society of Southern Africa and the South African Society of Surgeons in Training could provide the academic, structural and logistical support for us to adopt a similar model and encourage surgical trainee collaborative development in SA (Fig. 1)

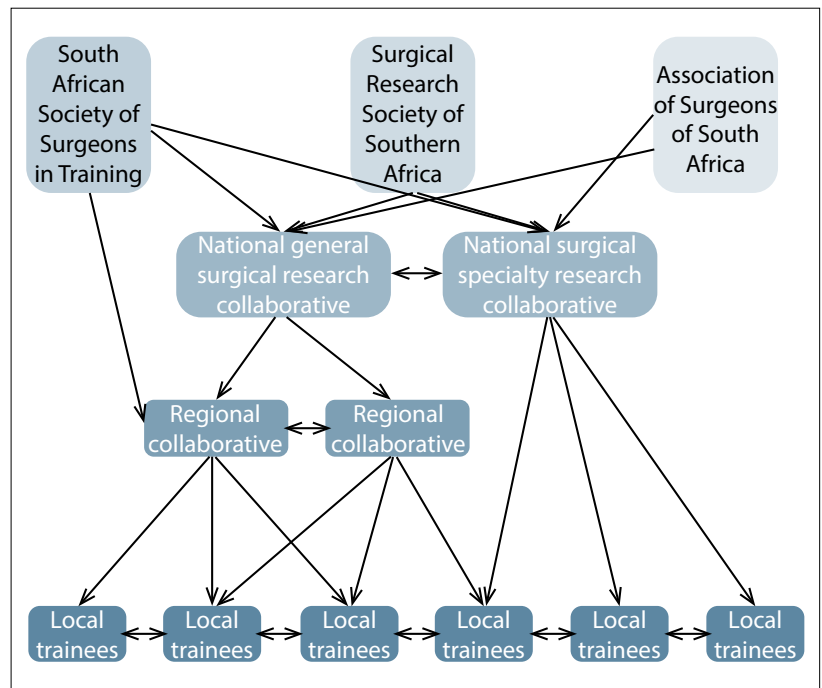

Fig. 1. A proposed model for organisation and communication of national research collaboratives in SA.

SA's position as neighbours to countries in SSA with the highest disease burdens and lowest audit and research capacity potentially allows inclusion of its local surgeons into a growing collaborative network. As in the UK, surgical collaboratives in SSA can seek to establish regional, national and international 'hub-and-spoke' models. This will allow universities and research centres to deliver research from several local partners, increasing strength, depth and value. As clinical studies and trials disseminate best clinical practice as part of their quality control, this should have rub-off benefits for patients beyond urban centres as networks develop.

\section{Conclusion}

The paucity of research in areas of greatest clinical need must be addressed urgently. We propose a model of collaboration in an era of information systems and emerging mobile health technology that has had significant success across the UK and has shown early encouraging results in SA. We foresee that recent examples of surgical research collaboratives in SA will continue to promote regional, national and international 'hub-and-spoke' models and ultimately increase the South-South collaboration that is urgently needed to diffuse the skills and knowledge required to address the unmet surgical need in SSA.

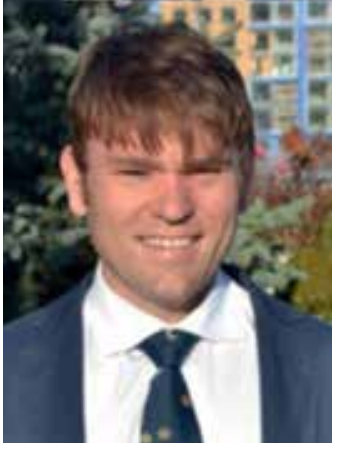

Richard Trafford Spence

Codman Center, Department of

General Surgery, Massachusetts

General Hospital, Boston, USA, and

Department of Surgery, Faculty of

Health Sciences, University of

Cape Town, South Africa

rtspence@mgh.harvard.edu

\section{Eugenio Panieri}

Department of Surgery, Faculty of Health Sciences, University of Cape Town, South Africa

\section{Sarah Louise Rayne}

Department of Surgery, Faculty of Health Sciences, University of the Witwatersrand, Johannesburg, South Africa

\section{Ewen Munro Harrison}

Clinical Surgery, University of Edinburgh and Royal Infirmary of Edinburgh, UK

\section{Aneel Amir Bhangu}

Academic Department of Surgery, University of Birmingham and Queen Elizabeth Hospital, Birmingham, UK

\section{James Edward Fitzgerald}

Department of General Surgery, Royal Free Hospital NHS,

Barnet Hospital Campus, London, UK

. Rottingen JA, Regmi S, Eide M, et al. Mapping of available health research and development data: What's there, what's missing, and what role is there for a global observatory? Lancet 2013;382(9900):12861307. [http://dx.doi.org/10.1016/S0140-6736(13)61046-6]

. Moran M, Guzman J, Ropars AL, et al. Neglected disease research and development: How much are we really spending? PLoS Med 2009;6(2):e30. [http://dx.doi.org/10.1371/journal.pmed.1000030]

3. Meara JG, Leather AJ, Hagander L, et al. Global Surgery 2030: Evidence and solutions for achieving health, welfare, and economic development. Lancet 2015;386(9993):569-624. [http://dx.doi.org/10.1016] S0140-6736(15)60160-X]

4. Gakwaya A, Kigula-Mugambe JB, Kavuma A, et al. Cancer of the breast: 5-year survival in a tertiary hospital in Uganda. Br J Cancer 2008;99(1):63-67. [http://dx.doi.org/10.1038/sj.bjc.6604435]

5. Pinkney TD, Calvert M, Bartlett DC, et al. Impact of wound edge protection devices on surgical Pinkney TD, Calvert M, Bartlett DC, et al. Impact of wound edge protection devices on surgical
site infection after laparotomy: Multicentre randomised controlled trial (ROSSINI Trial). BMJ 2013;347:f4305. [http://dx.doi.org/10.1136/bmi.f4305]

6. National Surgical Research Collaborative. Multicentre observational study of performance variation in provision and outcome of emergency appendicectomy. Br J Surg 2013;100(9):1240-1252. [http:// dx.doi.org/10.1002/bjs.9201

. Bhangu A, Kolias AG, Pinkney T, Hall NJ, Fitzgerald JE. Surgical research collaboratives in the UK. Lancet 2013;382(9898):1091-1092. [http://dx.doi.org/10.1016/S0140-6736(13)62013-9]

8. STARSurg Collaborative. Impact of postoperative non-steroidal anti-inflammatory drugs on adverse events after gastrointestinal surgery. Br J Surg 2014;101(11):1413-1423. [http://dx.doi.org/10.1002/bjs.9614]

9. Biccard BM, Madiba TE. The South African Surgical Outcomes Study: A 7-day prospective observational cohort study. S Afr Med J 2015;105(6):465-475. [http://dx.doi.org/10.7196/SAMJ.9435]

10. Bhangu A. Determining universal processes related to best outcome in emergency abdominal surgery: A multicentre, international, prospective cohort study. BMJ Open 2014;4(10):e006239. [http://dx.doi. org/10.1136/bmjopen-2014-006239]

11. Uthman OA, Wiysonge CS, Ota $\mathrm{MO}$, et al. Increasing the value of health research in the WHO African Region beyond 2015 - reflecting on the past, celebrating the present and building the future: A bibliometric analysis. BMJ Open 2015;5(3):e006340-2014-006340. [http://dx.doi.org/10.1136/ bmjopen-2014-006340]

S Afr Med J 2016;106(2):125-126. DOI:10.7196/SAMJ.2016.v106i2.10183 